\title{
PET Image Reconstruction with Kernel and Kernel Space Composite Regularizer
}

This paper was downloaded from TechRxiv (https://www.techrxiv.org).

LICENSE

CC BY 4.0

SUBMISSION DATE / POSTED DATE

$27-12-2021$ / 29-12-2021

\section{CITATION}

Guo, Shiyao; Sheng, Yuxia; Li, Shenpeng; Chai, Li; Zhang, Jingxin (2021): PET Image Reconstruction with Kernel and Kernel Space Composite Regularizer. TechRxiv. Preprint.

https://doi.org/10.36227/techrxiv.17694368.v1

$\mathrm{DOI}$

10.36227/techrxiv.17694368.v1 


\title{
PET Image Reconstruction with Kernel and Kernel Space Composite Regularizer
}

\author{
Shiyao Guo, Yuxia Sheng, Shenpeng Li, Li Chai and Jingxin Zhang
}

\begin{abstract}
Represented by the kernelized expectation maximization (KEM), the kernelized maximum-likelihood (ML) expectation maximization (EM) methods have recently gained prominence in PET image reconstruction, outperforming many previous state-of-the-art methods. But they are not immune to the problems of non-kernelized MLEM methods in potentially large reconstruction variance and high sensitivity to iteration number. Also, it is generally difficult to simultaneously reduce image variance and preserve image details using kernels. To solve these problems, this paper presents a novel regularized KEM (RKEM) method with a kernel space composite regularizer for PET image reconstruction. The composite regularizer consists of a convex kernel space graph regularizer that smoothes the kernel coefficients, a non-convex kernel space energy regularizer that enhances the coefficients' energy, and a composition constant that guarantees the convexity of composite regularizer. These kernel space regularizers are based on the theory of data manifold and graph regularization and can be constructed from different prior image data for simultaneous image variance reduction and image detail preservation. Using this kernel space composite regularizer and the technique of optimization transfer, a globally convergent iterative algorithm is derived for RKEM reconstruction. Tests and comparisons on the simulated and in vivo data are presented to validate and evaluate the proposed algorithm, and demonstrate its better performance and advantages over KEM and other conventional methods.
\end{abstract}

Index Terms-PET image reconstruction, regularized KEM, kernel space graph regularizer, kernel space energy regularizer.

\section{INTRODUCTION}

$\mathbf{K}$ ERNELIZED expectation maximization (KEM) method [1], [2] is a representative of the recent development of iterative methods for PET image reconstruction. It uses the prior data, such as MR anatomical images, to construct the kernel matrix and uses the maximum-likelihood (ML) expectation maximization (EM) algorithm in the kernel space to

S. Guo, Y. Sheng, L. Chai are with the Engineering Research Center of Metallurgical Automation and Measurement Technology, Wuhan University of Science and Technology, Wuhan, China. e-mails: wustguosy@foxmail.com; shengyuxia@wust.edu.cn; chaili@wust.edu.cn.

$\mathrm{S}$. $\mathrm{Li}$ is with Health and Biosecurity, the Australian e-Health Research Centre, CSIRO, Parkville, VIC 3052, Australia. e-mail: shenpeng.li@csiro.au.

J. Zhang is with the School of Science, Computing and Engineering Technologies, Swinburne University of Technology, Melbourne, Vic 3126, Australia. e-mail: jingxinzhang@swin.edu.au.

Research supported by National Natural Science Foundation of China (Grant No.s 61625305 and 61501337). iteratively estimate the kernel coefficients of the image, which in turn are mapped by the kernel matrix to the reconstructed image.

KEM has proven to perform better than conventional nonkernelized MLEM methods in enhancing the spatial and temporal resolution and quantification in static and dynamic PET imaging, with great potential in low dose PET imaging. Following these promising results, a number of kernelized methods have recently been presented for various PET image reconstruction problems, see eg [3]-[9].

Despite the advantages of kernelized MLEM methods, they may suffer the same problems of conventional MLEM methods. As discussed in [10], a well known problem of all ML methods in PET is ill-conditioning, which makes the solutions sensitive to small changes in the data so that the estimates are of high variance. This problem is shown in practice as the checkerboard effect of high spatial variance in the ML images for high iteration numbers of the EM algorithms. Because of the smoothing effect of kernel matrix, KEM's image variance is much lower than that of MLEM. But it still has the problem of increasingly high image variance at high iteration numbers, especially in low count imaging. This is observed in our experiments presented in Fig. 7 of Subsection III-D. A similar observation is also shown in [11], with a suggested fix to the problem by early stopping of iterations in the kernel-based EM method, which is a practical yet low performance fix to the same problem in conventional MLEM methods [10], [15].

For conventional MLEM methods, an effective high performance solution to the above problem is the regularized MLEM estimation [10], [12]-[15]. It constrains the MLEM estimates with the regularizers introduced as the priors of PET images. The properly chosen priors can significantly reduce the image variance at high iteration numbers and preserve the image details simultaneously [15]-[18]. To ensure the convergence of regularized MLEM estimation, a class of globally convergent iterative computation algorithms have been obtained using the optimization transfer technique [19]-[23]. However, these effective solutions have not been adopted in the kernelized methods yet, probably due to two reasons.

First, the kernels in kernelized methods can be tuned to significantly smooth out the image variance or to better preserve image details, so they have some regularization capability [2], [11]. This might have steered research attentions away from regularization to focus on finding better kernels and their better parameters for tradeoffs between reducing image variance and preserving image details. In fact, the kernels used in the 
kernelized methods of PET have very limited regularization capability, because they do not possess the key property of a regularizer detailed in [24]. Also, it is generally difficult to simultaneously reduce image variance and preserve image details by tuning kernels because they do not have sufficient freedom to achieve that.

Second, it is difficult to develop globally convergent algorithm for the kernelized methods with regularizers. As shown later in this paper, the optimization transfer is not directly applicable in this case.

Above facts strongly suggest that some explicit and effective regularizations are needed in the kernelized methods in order to reduce their reconstruction variance and sensitivity to iteration number and to preserve image details simultaneously. These have motivated us to develop in this paper a novel kernel space regularization method, called RKEM, for the KEM method to avoid its possibility of large reconstruction variance and high sensitivity to iteration number, and to enhance its image quality.

To overcome the difficulty in developing the globally convergent optimization algorithm for RKEM, we brow the ideas of manifold assumption and graph regularization from manifold learning [25] and sparse coding [26], [27] to develop a simple yet effective kernel space composite regularizer. Combining the kernel space composite regularizer with the optimization transfer technique [19], [22], [23], we present a globally convergent RKEM algorithm for PET image reconstruction. The novel ingredients of the kernel space composite regularizer set RKEM apart from the exiting kernelized methods and empower it with better performance.

We further use simulated and in vivo data to validate and evaluate the performance of RKEM, and demonstrate its better performance and advantages over the existing methods by comprehensive comparisons.

This paper is organized as follows. Section II presents our proposed method in detail. Section III presents the simulation studies and comparisons of the proposed method with the existing methods. Section IV presents the in vivo data testing results of the proposed method and compares with those of the existing methods. Discussions and Conclusions are presented in Sections V and VI, respectively.

\section{THEORY AND METHOD}

\section{A. PET Image Reconstruction with Regularized ML}

Let $\boldsymbol{y}=\left[y_{1} \cdots y_{i} \cdots y_{N}\right]^{T}$ be the PET projection data vector with the elements $y_{i}$ having the log-likelihood function [15]

$$
L(\boldsymbol{y} \mid \boldsymbol{x})=\sum_{i=1}^{N} y_{i} \log \bar{y}_{i}-\bar{y}_{i}-\log y_{i} !,
$$

where $N$ is the total number of lines of response and $\bar{y}_{i}$ is the expectation of $y_{i}$. The expectation of $\boldsymbol{y}, \overline{\boldsymbol{y}}=$ $\left[\bar{y}_{1} \cdots \bar{y}_{i} \cdots \bar{y}_{N}\right]^{T}$ is related to the unknown emission image $\boldsymbol{x}=\left[x_{1} \cdots x_{i} \cdots x_{M}\right]^{T}$ by the forward projection

$$
\overline{\boldsymbol{y}}=\boldsymbol{H} \boldsymbol{x}+\boldsymbol{r}
$$

where $x_{i}$ is the $i$ th voxel intensity of the image $\boldsymbol{x}, M$ is the total number of pixels, $\boldsymbol{H} \in \mathcal{R}^{N \times M}$ is the PET system matrix, and $\boldsymbol{r}=\left[r_{1} \cdots r_{i} \cdots r_{N}\right]^{T}$ is the expectation of random and scattered events. The image $\boldsymbol{x} \in \mathcal{R}^{M}$ here is the vectorization of a multi-dimensional PET image.

An estimate of the unknown image $\boldsymbol{x}$ can be obtained by the regularized maximum likelihood (RML), or equivalently maximum a posteriori (MAP), estimate [10], [15], [17], [23]

$$
\hat{\boldsymbol{x}}=\underset{\boldsymbol{x} \geq 0}{\arg \max }(L(\boldsymbol{y} \mid \boldsymbol{x})-\beta V(\boldsymbol{x}))
$$

where $L(\boldsymbol{y} \mid \boldsymbol{x})$ is as defined in (1), $\beta \geq 0$ is a regularization parameter, and $V(\boldsymbol{x})$ is a regularizer or prior function. When $\beta=0$, (3) reduces to the ML estimation, which, as is well known, generally produces noisy estimate with high variance and requires early termination when it is computed by EM iterations. If chosen properly, the regularizer $V(\boldsymbol{x})$ can effectively reduce MLEM's estimation variance and sensitivity to the iteration number, and hence improves the image quality of MLEM reconstruction [10], [15].

In this paper, we consider $V(\boldsymbol{x})$ as a composite of two regularizers

$$
V(\boldsymbol{x})=v_{1}(\boldsymbol{x})+\gamma v_{2}(\boldsymbol{x})
$$

where $\gamma \geq 0$ is a composition constant and $V(\boldsymbol{x})$ as a whole is a convex function.

\section{B. Kernelized PET Image Reconstruction with Regularized $M L$}

Following [2], the unknown emission image $\boldsymbol{x}$ can be presented in the kernel space as $\boldsymbol{x}=\boldsymbol{K} \boldsymbol{a}$, where $\boldsymbol{K} \in \mathcal{R}^{M \times M}$ is a kernel matrix and $\boldsymbol{a} \in \mathcal{R}^{M}$ is the coefficient vector of $\boldsymbol{x}$ under $\boldsymbol{K}$. The $i j$ th element of the kernel matrix $\boldsymbol{K}$ is given by $k_{i j}=\kappa\left(\tilde{\boldsymbol{x}}_{i}, \tilde{\boldsymbol{x}}_{j}\right)=\phi\left(\tilde{\boldsymbol{x}}_{i}\right)^{T} \phi\left(\tilde{\boldsymbol{x}}_{j}\right), i, j \in\{1,2, \cdots, M\}$, where $\kappa(\cdot, \cdot)$ is a kernel (function), $\tilde{\boldsymbol{x}}_{i}$ and $\tilde{\boldsymbol{x}}_{j}$ are respectively the data vectors for the $i$ th and $j$ th elements of $\boldsymbol{x}$ in the prior dataset $\tilde{\boldsymbol{X}}$ of $\boldsymbol{x}$ obtained from other sources, eg MR anatomical or prereconstructed PET images of the same (class of) subject/s, and $\phi(\cdot)$ is a nonlinear function mapping $\boldsymbol{x}$ to a higher dimensional feature space but needs not to be known explicitly.

The commonly used kernel in PET image reconstruction is the radial Gaussian kernel [2], [11]

$$
\kappa\left(\tilde{\boldsymbol{x}}_{i}, \tilde{\boldsymbol{x}}_{j}\right)=\exp \left(-\left\|\tilde{\boldsymbol{x}}_{i}-\tilde{\boldsymbol{x}}_{j}\right\|^{2} / 2 \sigma^{2}\right),
$$

where $\sigma$ is a kernel parameter, $\|\cdot\|$ is the 2-norm of a vector. For computation simplicity and performance improvement, the kernel matrix $\boldsymbol{K}$ in practice is often constructed using

$$
k_{i j}=\left\{\begin{array}{l}
\kappa\left(\tilde{\boldsymbol{x}}_{i}, \tilde{\boldsymbol{x}}_{j}\right), j \in \mathcal{J} \\
0, \text { otherwise }
\end{array}\right.
$$

where $\mathcal{J}$ is the neighborhood of the $i$ th element of emission image $\boldsymbol{x}$, with the neighborhood size $J$ defined as the $J$ nearest pixels of $\tilde{\boldsymbol{x}}_{i}$. Other methods such as $\tilde{\boldsymbol{x}}_{j} \in k N N$ (the $k$ nearest neighbor) of $\tilde{\boldsymbol{x}}_{i}$, measured by $\left\|\tilde{\boldsymbol{x}}_{i}-\tilde{\boldsymbol{x}}_{j}\right\|$, can also be used [2].

Using the kernel space image model $\boldsymbol{x}=\boldsymbol{K} \boldsymbol{a}$, we can convert the forward projection (2) and the log-posterior probability maximization (3) to their respective kernelized equivalents

$$
\begin{aligned}
& \overline{\boldsymbol{y}}=\boldsymbol{H} \boldsymbol{K} \boldsymbol{a}+\boldsymbol{r}, \\
& \hat{\boldsymbol{a}}=\arg \max _{\boldsymbol{a} \geq 0}(L(\boldsymbol{y} \mid \boldsymbol{K} \boldsymbol{a})-\beta V(\boldsymbol{K} \boldsymbol{a})),
\end{aligned}
$$


and reconstruct the unknown image $\boldsymbol{x}$ by

$$
\hat{\boldsymbol{x}}=\boldsymbol{K} \hat{\boldsymbol{a}} .
$$

As seen from above, the kernel matrix $\boldsymbol{K}$ serves as a basis (dictionary) of the underlining image $\boldsymbol{x}$ obtained from the high dimensional feature space of the nonlinearly transformed prior data $\phi\left(\tilde{\boldsymbol{x}}_{\boldsymbol{i}}\right)$, and $\boldsymbol{a}$ is the coefficient (code) of the image $\boldsymbol{x}$ under $\boldsymbol{K}$. The kernel matrix $\boldsymbol{K}$ limits the search of $\boldsymbol{x}$ to the linear combinations of its basis vectors. This may effectively improve image reconstruction if $\boldsymbol{K}$ is chosen properly [2].

The objective function of (8) is a kernelized RML. It reduces to the objective function of KEM [2] when $\beta=0$. Therefore, we call (8) with $\beta>0$ the regularized KEM (RKEM).

Exploiting the linear relation $\boldsymbol{x}=\boldsymbol{K} \boldsymbol{a}, \mathrm{KEM}$ uses standard EM algorithm to iteratively solve (8) with $\beta=0$ for $\hat{\boldsymbol{a}}$. Hence, KEM shares the same convergence property of conventional MLEM and also requires early stopping of EM iteration to avoid noisy reconstruction [11]. Similar to the function of $V(\boldsymbol{x})$ in the non-kernelized RML (3), the kernelized regularizer $V(\boldsymbol{K} \boldsymbol{a})$ in RKEM (8) is to reduce KEM's estimation variance and sensitivity to the iteration number and hence enhance its image quality.

\section{Kernel Space Composite Regularizer}

For the non-kernelized RML (3), its regularizer $V(\boldsymbol{x})$ as must be convex in order to have a unique global maxima [10], [28], and iterative optimization is generally needed for its solution. To ensure the convergence of iterative optimization, the optimization transfer method [19] is often used to derive the convergent iterative algorithm, see eg [22], [23]. Since the optimizations (3) and (8) are equivalent due to $\boldsymbol{x}=$ $\boldsymbol{K} \boldsymbol{a}$, the RKEM (8) with $\boldsymbol{x}$ replaced by $\boldsymbol{K} \boldsymbol{a}$ also needs a convex composite regularizer and globally convergent iterative algorithm for its optimization. Construction of regularizer is crucial to derive such algorithm.

A commonly used regularizer in the literature for the nonkernelized RML (3) is the quadratic image smoothing function [10], [15], [23] (corresponding to $\gamma=0$ and $V(\boldsymbol{x})=v_{1}(\boldsymbol{x})=$ $v(\boldsymbol{x})$ in (4))

$$
v(\boldsymbol{x})=\frac{1}{2} \sum_{i=1}^{M} \sum_{j \in \mathcal{J}} \psi_{i j}\left(x_{i}-x_{j}\right)^{2}=\boldsymbol{x}^{T} \boldsymbol{L}_{\Psi} \boldsymbol{x}
$$

where $\psi_{i j}=\psi_{j i} \geq 0$ are weights, $\boldsymbol{L}_{\Psi}=\boldsymbol{D}-\boldsymbol{\Psi}$ is a Laplacian matrix with the degree matrix $\boldsymbol{D}=\operatorname{diag}\left[\left(\sum_{j \in \mathcal{J}} \psi_{1 j}\right)\right.$ $\left.\cdots\left(\sum_{j \in \mathcal{J}} \psi_{M j}\right)\right]$ and the weight matrix $\boldsymbol{\Psi}$ consisting of $\psi_{i j}$ for $i \in\{1,2, \cdots, M\}, j \in \mathcal{J}$, and 0 otherwise. It has been used in eg [10], [23] to derive convergent iterative algorithms for the non-kernelized RML (3). A key technique in deriving these algorithms is the so called surrogate functions. They are used to substitute the likelihood and regularizer functions in (3) and make the resulting surrogate objective function separable for each $x_{i}, i \in\{1,2, \cdots, M\}$, [10], [19].

However, this technique is not directly applicable to the RKEM (8) where $\boldsymbol{x}$ is replaced by $\boldsymbol{K} \boldsymbol{a}$ and $\boldsymbol{a}$ is used as the optimizer. In this case $v(\boldsymbol{x})$ becomes

$$
\begin{aligned}
v(\boldsymbol{K} \boldsymbol{a}) & =\frac{1}{2} \sum_{i=1}^{M} \sum_{j \in \mathcal{J}} \psi_{i j}\left(\boldsymbol{K}_{i} \boldsymbol{a}-\boldsymbol{K}_{j} \boldsymbol{a}\right)^{2} \\
& =\frac{1}{2} \sum_{i=1}^{M} \sum_{j \in \mathcal{J}} \psi_{i j}\left(\sum_{l=1}^{M} k_{i l} a_{l}-\sum_{l=1}^{M} k_{j l} a_{l}\right)^{2}
\end{aligned}
$$

where $\boldsymbol{K}_{i}=\left[k_{i 1} \cdots k_{i M}\right]$ and $\boldsymbol{K}_{j}=\left[k_{j 1} \cdots k_{j M}\right]$ are the $i$ th and $j$ th rows of $\boldsymbol{K}, \boldsymbol{K}_{i} \boldsymbol{a}=x_{i}$ and $\boldsymbol{K}_{j} \boldsymbol{a}=x_{j}$. Since $\boldsymbol{K}$ is generally a band matrix with multiple nonzero elements in each row, $x_{i}=\boldsymbol{K}_{i} \boldsymbol{a}$ and $x_{j}=\boldsymbol{K}_{j} \boldsymbol{a}$ both contain multiple $a_{l}$ 's, $l \in\{1,2, \cdots, M\}$. Simply replacing $x_{i}$ and $x_{j}$ by $\boldsymbol{K}_{i} \boldsymbol{a}$ and $\boldsymbol{K}_{j} \boldsymbol{a}$ in the existing surrogate functions of $v(\boldsymbol{x})$ (see eg [10], [23]) cannot make the surrogate objective function of (8) separable for each optimization variable $a_{i}$. To overcome this difficulty, we propose below a novel kernel space graph regularizer.

First, we construct a kernel matrix $\boldsymbol{K}^{O} \in \mathcal{R}^{M \times M}$ using the prior image data $\tilde{\boldsymbol{x}}_{i}^{O}$ and $\tilde{\boldsymbol{x}}_{j}^{O}$ and the same kernel and kernel parameters (5)-(6) as those for the kernel matrix $\boldsymbol{K}$ in the RKEM reconstruction (7)-(9). The prior image data $\tilde{\boldsymbol{x}}_{i}^{O}$ and $\tilde{\boldsymbol{x}}_{j}^{O}$ here can be different from or the same as the prior image data $\tilde{\boldsymbol{x}}_{i}$ and $\tilde{\boldsymbol{x}}_{j}$ for constructing the kernel matrix $\boldsymbol{K}$. For example, $\tilde{\boldsymbol{x}}_{i}^{O}$ and $\tilde{\boldsymbol{x}}_{j}^{O}$ can be from pre-reconstructed PET images, and $\tilde{\boldsymbol{x}}_{i}$ and $\tilde{\boldsymbol{x}}_{j}$ from MR anatomical images. When $\tilde{\boldsymbol{x}}_{i}^{O}$ and $\tilde{\boldsymbol{x}}_{j}^{O}$ are the same as $\tilde{\boldsymbol{x}}_{i}$ and $\tilde{\boldsymbol{x}}_{j}, \boldsymbol{K}^{O}=\boldsymbol{K}$.

Next, we construct a doubly normalized Laplacian matrix

$$
\boldsymbol{L}=\boldsymbol{I}-\boldsymbol{W}, \quad \boldsymbol{W}=\boldsymbol{C}^{(-1 / 2)} \boldsymbol{K}^{O} \boldsymbol{C}^{(-1 / 2)}
$$

where $\boldsymbol{I}$ is identity matrix and $\boldsymbol{C}$ is a diagonal scaling matrix obtained by applying Sinkhorn matrix balancing procedure [29] to the kernel matrix $\boldsymbol{K}^{O}$. This Laplacian matrix $\boldsymbol{L}$ defines a graph of the image $\boldsymbol{x}$, with the adjacency matrix $\boldsymbol{W}$. The adjacency matrix $\boldsymbol{W}$ constructed above is a symmetric nonnegative doubly stochastic matrix, with each row and column summing to 1 , representing the transition probabilities of pixels $x_{i}$ 's along the graph [30].

With $\boldsymbol{L}$ obtained from (11), we define a Laplacian quadratic of the kernel coefficient $\boldsymbol{a}$

$$
v_{1}(\boldsymbol{a})=\boldsymbol{a}^{T} \boldsymbol{L} \boldsymbol{a}=\frac{1}{2} \sum_{i=1}^{M} \sum_{j \in \mathcal{J}} w_{i j}\left(a_{j}-a_{i}\right)^{2}
$$

and use it as a regularizer for the RKEM (8), where $w_{i j}$ is the $i j$ th element of $\boldsymbol{W}$ given in (11). This regularizer is the same form smoothing function as that of (10), but it is in the kernel coefficients $a_{i}$, instead of the image pixels $x_{i}=\boldsymbol{K}_{i} \boldsymbol{a}$, and has the weights $w_{i j} \geq 0$ specified by (11). As shown in Subsection II-D, replacing $x_{i}$ with $a_{i}$ in the surrogate functions previously used for the $v(\boldsymbol{x})$ in (10), see eg [10], [23], we can easily obtain a surrogate function for the regularizer $v_{1}(\boldsymbol{a})$ and use it to separate each coefficient $a_{i}, i \in\{1,2, \cdots, M\}$, in the surrogate objective function of the RKEM (8).

The use of regularizer $v_{1}(\boldsymbol{a})=\boldsymbol{a}^{T} \boldsymbol{L} \boldsymbol{a}$ is based on the fact that high dimensional data in real world, such as that of an image, generally lie on low-dimensional manifolds, and a manifold can be represented by a graph defined by 
the Laplacian matrix constructed from the data [25]. If two data points are close in the intrinsic data manifold, then their representations in any other basis are also close. This is the so called manifold assumption that has been used in manifold learning and dimensionality reduction [25], clustering [31], [32], semisupervised learning [33], [34] and sparse coding [26], [27] to develop various effective methods. It has been shown in [26], [27] that a signal $\boldsymbol{x}$ and its coefficient (code) $\boldsymbol{a}$ under a basis (dictionary) share the same smoothness property in the manifold of $\boldsymbol{x}$ represented by a graph. Using the Laplacian matrix $\boldsymbol{L}$ of this graph constructed from the data of $\boldsymbol{x}$, the Laplacian quadratic $\boldsymbol{a}^{T} \boldsymbol{L} \boldsymbol{a}$ can be used as a regularizer to control the smoothness of $\boldsymbol{x}$, instead of using $\boldsymbol{x}^{T} \boldsymbol{L} \boldsymbol{x}$. This method is borrowed here for the RKEM reconstruction of PET image. Because $v_{1}(\boldsymbol{a})=\boldsymbol{a}^{T} \boldsymbol{L} \boldsymbol{a}$ is derived from a graph and is in the kernel coefficients $\boldsymbol{a}$, we call it the kernel space graph regularizer.

Similar to its image space counterpart $\boldsymbol{x}^{T} \boldsymbol{L} \boldsymbol{x}$, the regularizer $\boldsymbol{a}^{T} \boldsymbol{L} \boldsymbol{a}$ may over-smooth the kernel coefficient $\boldsymbol{a}$, which in turn may over-smooth the edges and small objects in the reconstructed image. To mitigate this problem, we introduce another kernel space regularizer

$$
v_{2}(\boldsymbol{a})=-\boldsymbol{a}^{T} \boldsymbol{W}_{D} \boldsymbol{a}=-\sum_{i=1}^{M} w_{i i} a_{i}^{2}
$$

where $w_{i i}, i=1,2, \cdots, M$, are the diagonal elements of the adjacency matrix $\boldsymbol{W}$ constructed in (11) and $\boldsymbol{W}_{D}$ is the diagonal matrix consisting of $w_{i i}$ 's.

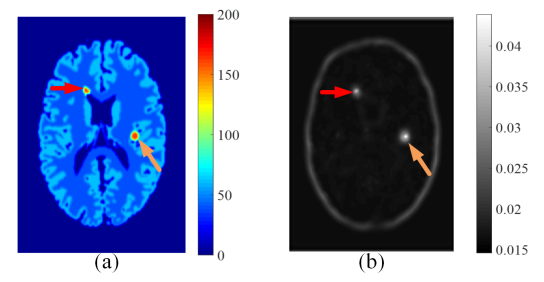

Fig. 1. (a) Simulated PET image with two lesions. (b) Image of corresponding $\boldsymbol{w}_{\boldsymbol{i} i}$ 's.

Since $v_{2}(\boldsymbol{a})$ is negative valued, it promotes the total energy of $\boldsymbol{a}$ when it is used as a regularizer in the RKEM (8). The promotion of each $a_{i}$, the $i$ th element of $\boldsymbol{a}$, is determined by its weight $w_{i i}$. By the construction of $\boldsymbol{W}, w_{i i} \geq 0$ is the selfloop transition probability of the pixel $x_{i}$ on the graph. For the $x_{i}$ 's at the non-smooth areas of edges and small objects in the image, their intensities are higher than other pixels' and hence their corresponding self-loop transition probability $w_{i i}$ 's are also higher for them to remain at their locations during the transitions. This is demonstrated in Fig. 1, where (a) shows a simulated PET image with $M$ pixels and two lesions of different sizes and intensities, which is used to construct the adjacency matrix $\boldsymbol{W}$ of (11), and (b) shows the image consisting of the diagonal elements, $w_{i i}, i=1,2, \cdots, M$, of the $\boldsymbol{W}$. As seen from these images, the locations and intensities of larger (brighter) $w_{i i}$ 's coincide with those of the lesions in the simulated PET image. Since $a_{i}$ 's and $x_{i}$ 's share the same smoothness (or non-smoothness) property in the manifold of $\boldsymbol{x}$, we use $w_{i i}$ as the weight of $a_{i}^{2}$ in the regularizer $v_{2}(\boldsymbol{a})$. The larger the weight $w_{i i}$, the more enhancement of $a_{i}^{2}$ and the more its contribution to the total energy of $\boldsymbol{a}$. This in turn results in a brighter $x_{i}$ with higher energy at the corresponding location of the reconstructed image. Hence, we call $v_{2}(\boldsymbol{a})$ the kernel space energy regularizer in the sequel.

With the regularizers $v_{1}(\boldsymbol{a})$ and $v_{2}(\boldsymbol{a})$ introduced in (11) and (13), we define a kernel space composite regularizer $V(\boldsymbol{a})$ and use it as $V(\boldsymbol{K} \boldsymbol{a})$ in the RKEM objective function of (8).

$$
\begin{aligned}
V(\boldsymbol{a}) & =v_{1}(\boldsymbol{a})+\gamma v_{2}(\boldsymbol{a}) \\
& =\boldsymbol{a}^{T}\left(\boldsymbol{L}-\gamma \boldsymbol{W}_{D}\right) \boldsymbol{a} \\
& =\frac{1}{2} \sum_{i=1}^{M} \sum_{j \in \mathcal{J}} w_{i j}\left(a_{i}-a_{j}\right)^{2}-\gamma \sum_{i=1}^{M} w_{i i} a_{i}^{2} \\
& =\sum_{i=1}^{M} \Gamma\left(a_{i}\right),
\end{aligned}
$$

where $\gamma>0$ is a composition constant and $\Gamma\left(a_{i}\right)=$ $\frac{1}{2} \sum_{j \in \mathcal{J}} w_{i j}\left(a_{i}-a_{j}\right)^{2}-\gamma w_{i i} a_{i}^{2}$.

A sufficient condition for the convexity of $V(\boldsymbol{a})$ is to make $\Gamma\left(a_{i}\right)$ convex for all $i$, and the necessary and sufficient condition to achieve this is [35]

$$
\frac{\partial^{2} \Gamma\left(a_{i}\right)}{\partial a_{i}^{2}}=\sum_{j \in \mathcal{J}} w_{i j}-2 \gamma w_{i i} \geq 0, \forall i \in\{1,2, \cdots, M\} .
$$

As $\boldsymbol{W}$ is doubly stochastic with $\sum_{j \in \mathcal{J}} w_{i j}=1$ [30], (15) amounts to $\gamma \leq \frac{1}{2 w_{i i}}, \forall i \in\{1,2, \cdots, M\}$. To satisfy this condition, it suffices to set the composition constant

$$
\gamma=\frac{1}{2 w_{\max }},
$$

where $w_{\max }=\max \left\{w_{i i}, i=1,2, \cdots, M\right\}$.

Replacing $\boldsymbol{a}$ with $\boldsymbol{x}$ in the kernel space composite regularizer $V(\boldsymbol{a})$ we can also obtain an image space composite regularizer

$$
\begin{aligned}
V(\boldsymbol{x}) & =v_{1}(\boldsymbol{x})+\gamma v_{2}(\boldsymbol{x}) \\
& =\boldsymbol{x}^{T}\left(\boldsymbol{L}-\gamma \boldsymbol{W}_{D}\right) \boldsymbol{x} \\
& =\frac{1}{2} \sum_{i=1}^{M} \sum_{j \in \mathcal{J}} w_{i j}\left(x_{i}-x_{j}\right)^{2}-\gamma \sum_{i=1}^{M} w_{i i} x_{i}^{2}
\end{aligned}
$$

for the non-kernelized RML optimization (3). Notice that $V(\boldsymbol{a})$ and $V(\boldsymbol{x})$ have the same $\boldsymbol{L}$ and $\boldsymbol{W}_{D}$, and $\boldsymbol{a}$ and $\boldsymbol{x}$ share the same smoothness properties in the manifold of $\boldsymbol{x}$. Hence, the setting of $\gamma$ given in (16) and all the above discussions on $V(\boldsymbol{a})$ carry over to $V(\boldsymbol{x})$. With the weights $w_{i j}$ specified by (11) and the negative valued $v_{2}(\boldsymbol{x})$ specified by (13) and (16), this image space composite regularizer $V(\boldsymbol{x})$ differs from those in the previous literature for the non-kernelized RML reconstruction, eg [10], [15], [23], and has better performance, see Section III for demonstration.

\section{Kernelized Surrogate Objective Function and Convergent Iterative Optimization}

With $V(\boldsymbol{a})$ defined above, we now derive a convergent iterative algorithm for the RKEM (8) using the optimization 
transfer method [10], [21]. To do this, we denote $Q\left(\boldsymbol{a} ; \boldsymbol{a}^{n}\right)$ the kernelized surrogate objective function satisfying

$$
\begin{gathered}
Q\left(\boldsymbol{a} ; \boldsymbol{a}^{n}\right)-Q\left(\boldsymbol{a}^{n} ; \boldsymbol{a}^{n}\right) \leq \Phi(\boldsymbol{a})-\Phi\left(\boldsymbol{a}^{n}\right), \\
\nabla Q\left(\boldsymbol{a}^{n} ; \boldsymbol{a}^{n}\right)=\nabla \Phi\left(\boldsymbol{a}^{n}\right),
\end{gathered}
$$

where $\nabla$ is the gradient operator, $\Phi(\boldsymbol{a})=L(\boldsymbol{y} \mid \boldsymbol{K} \boldsymbol{a})-\beta V(\boldsymbol{a})$ is the original objective function of (8) with the regularizer $V(\boldsymbol{a})$ defined in (14), and $\boldsymbol{a}^{n}$ is the estimated kernel coefficient at the $n$th iteration.

The purpose of $Q\left(\boldsymbol{a} ; \boldsymbol{a}^{n}\right)$ is to substitute $\Phi(\boldsymbol{a})$ and convert (8) to $\hat{\boldsymbol{a}}=\arg \max _{\boldsymbol{a}>0} Q\left(\boldsymbol{a} ; \boldsymbol{a}^{n}\right)$. It then follows from the conditions (18)-(19) that solving this equivalent problem iteratively for the globally optimal solution is equivalent to solving (8) for its globally optimal solution. In accordance with $\Phi(\boldsymbol{a})$, we define

$$
Q\left(\boldsymbol{a} ; \boldsymbol{a}^{n}\right)=Q_{L}\left(\boldsymbol{a} ; \boldsymbol{a}^{n}\right)-\beta Q_{V}\left(\boldsymbol{a} ; \boldsymbol{a}^{n}\right),
$$

where $Q_{L}\left(\boldsymbol{a} ; \boldsymbol{a}^{n}\right)$ and $Q_{V}\left(\boldsymbol{a} ; \boldsymbol{a}^{n}\right)$ are respectively the surrogate functions for $L(\boldsymbol{y} \mid \boldsymbol{K} \boldsymbol{a})$ and $V(\boldsymbol{a})$.

For the kernelized likelihood function $L(\boldsymbol{y} \mid \boldsymbol{K} \boldsymbol{a})$, we use the surrogate function $Q_{L}\left(\boldsymbol{x} ; \boldsymbol{x}^{n}\right)$ of [21], [23], with $\boldsymbol{x}$ substituted by $\boldsymbol{K} \boldsymbol{a}$ and $\boldsymbol{K}$ absorbed into $Q_{L}(\cdot)$, to obtain the surrogate function

$$
Q_{L}\left(\boldsymbol{a} ; \boldsymbol{a}^{n}\right)=\sum_{i=1}^{M} F_{i}\left(\hat{a}_{i, E M}^{n+1} \log a_{i}-a_{i}\right),
$$

where $F_{i}=\sum_{k=1}^{N} G_{k i}, G_{k i}$ is the $k i$ th element of $\boldsymbol{G}=$ $\boldsymbol{H} \boldsymbol{K} \in R^{N \times M}, \hat{a}_{i, E M}^{n+1}$ is computed by

$$
\hat{a}_{i, E M}^{n+1}=\frac{a_{i}^{n}}{F_{i}} \sum_{k=1}^{N} G_{k i} \frac{y_{k}}{\left[\boldsymbol{G a}^{n}\right]_{k}+r_{k}},
$$

and $\left[\boldsymbol{G a}^{n}\right]_{k}$ is the $k$ th element of $\boldsymbol{G} \boldsymbol{a}^{n}$. It is easy to prove that $Q_{L}\left(\boldsymbol{a} ; \boldsymbol{a}^{n}\right)-Q_{L}\left(\boldsymbol{a}^{n} ; \boldsymbol{a}^{n}\right) \leq L(\boldsymbol{y} ; \boldsymbol{a})-L\left(\boldsymbol{y} ; \boldsymbol{a}^{n}\right)$ and $\nabla Q_{L}\left(\boldsymbol{a}^{n} ; \boldsymbol{a}^{n}\right)=\nabla L\left(\boldsymbol{y} ; \boldsymbol{a}^{n}\right)$.

For the kernel space composite regularizer $V(\boldsymbol{a})$ defined in (14), we only need to decouple $v_{1}(\boldsymbol{a})$ as $a_{i}$ 's in $v_{2}(\boldsymbol{a})$ are already decoupled. Using De Pierros decoupling rule [19], [23]

$$
\left(a_{i}-a_{j}\right)^{2} \leq 2\left(a_{i}-\frac{a_{i}^{n}+a_{j}^{n}}{2}\right)^{2}+2\left(a_{j}-\frac{a_{i}^{n}+a_{j}^{n}}{2}\right)^{2}
$$

we define the surrogate function $Q_{V}\left(\boldsymbol{a} ; \boldsymbol{a}^{n}\right)$ for $V(\boldsymbol{a})$

$$
\begin{aligned}
Q_{V}\left(\boldsymbol{a} ; \boldsymbol{a}^{n}\right)= & \sum_{i}^{M} \sum_{j \in \mathcal{J}} w_{i j}\left(\left(a_{i}-\frac{a_{i}^{n}+a_{j}^{n}}{2}\right)^{2}+\right. \\
& \left.\left(a_{j}-\frac{a_{i}^{n}+a_{j}^{n}}{2}\right)^{2}\right)-\gamma \sum_{i=1}^{M} w_{i i} a_{i}^{2},
\end{aligned}
$$

which satisfies $Q_{V}\left(\boldsymbol{a} ; \boldsymbol{a}^{n}\right)-Q_{V}\left(\boldsymbol{a}^{n} ; \boldsymbol{a}^{n}\right) \geq V(\boldsymbol{a})-V\left(\boldsymbol{a}^{n}\right)$ and $\nabla Q_{V}\left(\boldsymbol{a}^{n} ; \boldsymbol{a}^{n}\right)=\nabla V\left(\boldsymbol{a}^{n}\right)$.

With $Q_{L}\left(\boldsymbol{a} ; \boldsymbol{a}^{n}\right)$ and $Q_{V}\left(\boldsymbol{a} ; \boldsymbol{a}^{n}\right)$ introduced in (21)-(23), it is easy to show that the surrogate objective function $Q\left(\boldsymbol{a} ; \boldsymbol{a}^{n}\right)$ defined in (20) satisfies the conditions (18)-(19). Hence, we can perform the RKEM reconstruction (8)-(9) using

$$
\boldsymbol{a}^{n+1}=\arg \max _{\boldsymbol{a} \geq 0}\left(Q_{L}\left(\boldsymbol{a} ; \boldsymbol{a}^{n}\right)-\beta Q_{V}\left(\boldsymbol{a} ; \boldsymbol{a}^{n}\right)\right),
$$

$$
\hat{\boldsymbol{x}}=\boldsymbol{K} \boldsymbol{a}^{n+1} .
$$

As seen from (21)-(23), the optimization (24) is equivalent to a sequence of $M$ optimizations

$$
\begin{aligned}
a_{i}^{n+1}= & \underset{a_{i} \geq 0}{\arg \max } q_{i}\left(a_{i}\right), i=1,2, \cdots, M, \\
q_{i}\left(a_{i}\right)= & F_{i}\left(\hat{a}_{i, E M}^{n+1} \log a_{i}-a_{i}\right)- \\
& \beta \sum_{j \in \mathcal{J}} w_{i j}\left(\left(a_{i}-\frac{a_{i}^{n}+a_{j}^{n}}{2}\right)^{2}+\right. \\
& \left.\left(a_{j}-\frac{a_{i}^{n}+a_{j}^{n}}{2}\right)^{2}\right)+\beta \gamma w_{i i} a_{i}^{2},
\end{aligned}
$$

which can be solved by iterative calculation of $a_{i}^{n+1}$ for $i=$ $1,2, \cdots, M$, sequentially.

The estimation of $a_{i}$ at the iteration $n+1$ can be calculated by

$$
a_{i}^{n+1}=\frac{2 A_{i}}{B_{i}+\sqrt{B_{i}^{2}+4 A_{i} C_{i}}}
$$

where $A_{i}=F_{i} \hat{a}_{i, E M}^{n+1}, B_{i}=\left(F_{i}-\beta \sum_{j \in \mathcal{J}} w_{i j}\left(a_{i}^{n}+a_{j}^{n}\right)\right)$, $C_{i}=2 \beta\left(\sum_{j \in \mathcal{J}} w_{i j}-\gamma w_{i i}\right)=2 \beta\left(1-\gamma w_{i i}\right)$, all the variables in $A_{i}, B_{i}$ and $C_{i}$ are as given in (21)-(23), and $\gamma=\frac{1}{2 w_{\max }}$ is as given in (16).

The $a_{i}^{n+1}$ 's thus estimated give rise to the coefficient vector $\boldsymbol{a}^{n+1}$ that is used in (25) to obtain the estimated image $\hat{\boldsymbol{x}}$.

Similar to the optimization transfer algorithm of [23] for the non-kernelized image space RML optimization, the iterative algorithm above guarantees a monotonic convergence to the global solution of RKEM (8). When $\beta=0$, it reduces to the KEM algorithm [2] without regularization.

\section{E. Proposed Method}

Algorithm 1 summarizes the proposed RKEM method in pseudo-code, where the prior image data $\tilde{\boldsymbol{x}}^{O}$ is assumed to be a PET image pre-reconstructed by MLEM with Gaussian filtering. It can be replaced by other priors such as the MR image $\tilde{\boldsymbol{x}}$ if needed.

Letting $\boldsymbol{K}=\boldsymbol{I}$ and replacing $\boldsymbol{a}$ with $\boldsymbol{x}$, Algorithm 1 also gives a globally convergent iterative algorithm for the nonkernelized RML optimization (3) with the new image space composite regularizer $V(\boldsymbol{x})$ defined in (17). For brevity, we call this algorithm the RMLEM algorithm in the sequel.

\section{Simulation StUdies}

To investigate its behavior and performance, we have applied the proposed RKEM algorithm to the simulated low count and high count sinogram data, and compared its performance in PET image reconstruction with that of the existing PET image reconstruction algorithms. Since the KEM algorithm [1], [2] has proven to outperform many state-of-the-art PET image reconstruction algorithms using anatomical priors, we have mainly compared RKEM with KEM, RMLEM (defined in Subsection II-E), and MLEM-BW (MLEM with Bowsher regularizer) [36]. We have also compared RKEM with commonly used MLEM plus Gaussian filtering (MLEM+F) 


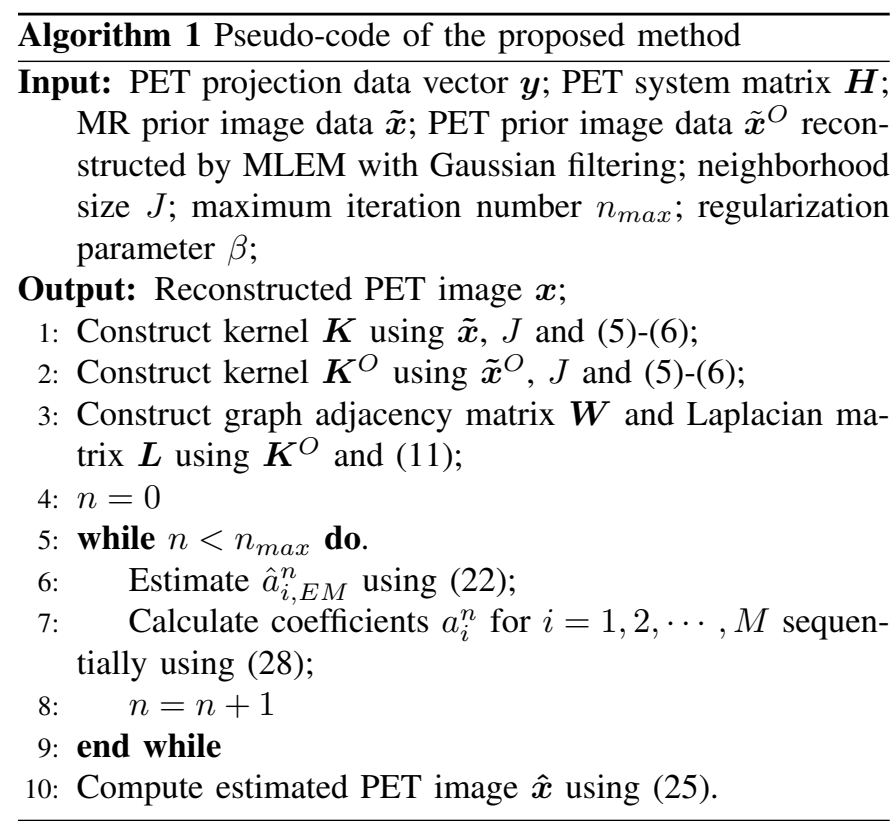

to show some common problems of PET image reconstruction methods that are based on the un-regularized MLEM.

As shown in [1], KEM with its kernel matrix $\boldsymbol{K}$ constructed using MR prior image and large neighborhood size $J$ and kernel parameter $\sigma$ may perform poorly on PET-only lesion. This is because the MR prior image does not have the information to distinguish the lesion from surrounding tissues. Reducing $J$ and $\sigma$ may enhance the lesion, but it may also make image reconstruction noisy. Tuning $J$ and $\sigma$ to achieve simultaneously the best lesion preservation and the best noise suppression is generally difficult for KEM. The kernel space graph regularizer $v_{1}(\boldsymbol{a})$ and the kernel space composite regularizer $V(\boldsymbol{a})$ in the proposed RKEM provide an effective technical means to overcome this difficulty.

To demonstrate these, we have implemented and compared in our experiments two versions of KEM and RKEM: KEM1, with smaller $J$ and $\sigma$ to show KEM's best performance in preserving PET-only lesion; KEM2, with larger $J$ and $\sigma$ to show KEM's best performance in noise suppression; RKEM-S, with KEM1 regularized by the kernel space graph regularizer $v_{1}(\boldsymbol{a})$ having the $J$ and $\sigma$ of $\boldsymbol{K}^{O}$ the same as those of KEM1, to show the effect of $v_{1}(\boldsymbol{a})$ on the noise suppression of KEM; RKEM, with KEM2 regularized by the kernel space composite regularizer $V(\boldsymbol{a})$ having the $J$ and $\sigma$ of $\boldsymbol{K}^{O}$ the same as those of KEM2, to show the effect of full version RKEM with $V(\boldsymbol{a})$ on simultaneous enhancement of PET-only lesion and noise suppression. We have not implemented and compared RKEM with only the kernel space energy regularizer $v_{2}(\boldsymbol{\alpha})$ defined in (13), because it is a nonconvex function and cannot be used alone for convergent iterative optimization.

\section{A. PET and Anatomical MRI Data Simulation}

The same method as that of [2] was used to generate 2D PET activity images. The 2D anatomical model from BrainWeb database consisting of 10 tissue classes was used to simulate the corresponding PET images [37], [38]. The regional time activities at 50 minutes were assigned to different brain regions to obtain a PET activity image of size $217 \times 181$, and two simulated lesions were added to this image. The resulting image was smoothed by a Gaussian filter with standard deviation $1 \mathrm{~mm}$ to simulate the point spread function of a typical PET scanner. The smoothed image as shown in Fig. 2(a) was used as the reference PET image, with the two simulated lesions shown respectively in Lesion 1 and Lesion 2 areas. A matching T1-weighted 2D MR image of size $217 \times 181$ and pixel resolution $1 \times 1 \mathrm{~mm}^{2}$ was generated from BrainWeb database, with a single simulated lesion at the same location as that of Lesion 1 in the reference PET image. To simulate the real scan process and test the robustness of different methods, noise was added to this MR image by BrainWeb. The MR image thus generated was used as the MR prior image data $\tilde{\boldsymbol{x}}$ shown in Fig. 2(b). In the sequel, we call Lesion 1 the PET-MR lesion and Lesion 2 the PET-only lesion.

The reference PET image was forward projected to generate noiseless sinograms using the PET system matrix generated by Fessler toolbox (https://web.eecs.umich.edu/ fessler/code/). The generated noiseless sinograms contained 249 radial bins and 210 angular projections, and were converted to noisy sinograms with different count levels by introducing the Poisson noise with the expected total numbers of events set to $1133 \mathrm{k}$ and $483 \mathrm{k}$ respectively. Attenuation maps, mean scatters and random events were included in all reconstruction methods to obtain quantitative images. Ten noisy realizations were simulated and each was reconstructed independently for comparison.

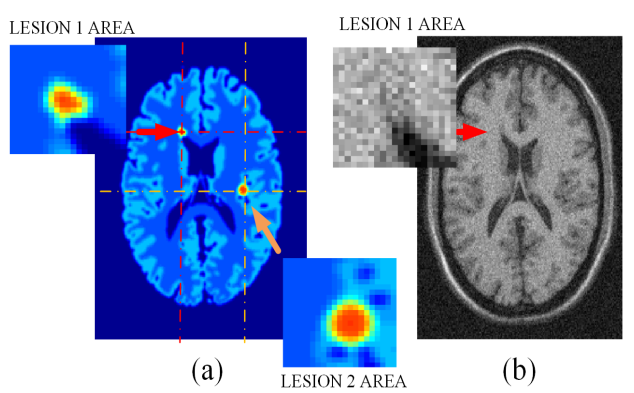

Fig. 2. (a) Simulated reference PET image with Lesion 1 and Lesion 2. (b) Simulated MR prior image with a lesion at the same location as that of Lesion 1 in (a).

\section{B. Algorithms Setup and Performance Metrics}

The parameters for KEM1, KEM2, RKEM-S and RKEM discussed above were as follows. KEM1: $J=11$ and $\sigma=$ 0.15 , giving KEM's best preservation of PET-only lesion. KEM2: $J=19$ and $\sigma=0.25$, giving KEM's best noise suppression. RKEM-S: the same $J=11$ and $\sigma=0.15$ as those of KEM1 in $\boldsymbol{K}^{O}$ construction. RKEM: the same $J=19$ and $\sigma=0.25$ as those of KEM2 in $\boldsymbol{K}^{O}$ construction.

For KEM1 and KEM2, the MR prior image shown in Fig. 2(b) was used to construct their kernel matrix $\boldsymbol{K}$. For RKEM$\mathrm{S}$ and RKEM, pre-reconstructed PET image was used to construct the kernel matrix $\boldsymbol{K}^{O}$ for the kernel space graph and composite regularizers $v_{1}(\boldsymbol{a})$ and $v(\boldsymbol{a})$. The pre-reconstructed PET image was obtained from the noisy PET sinogram data 
described in Subsection III-A using 30 iterations of MLEM, followed by Gaussian filtering.

The regularization parameter of RKEM-S and RKEM used in (28) was tuned to $\beta=4 \times 10^{-3}$ for both the low count and high count image reconstructions. The same $\beta$ values were used for RMLEM. The regularization parameter of MLMEBW was tuned to 0.003 .

For fair comparison, the above parameters and the total iteration numbers were obtained from tuning the compared methods separately to achieve their respective best tradeoff between the whole image mean square error (MSE) and the lesion MSE in image reconstruction.

The mean square error, $\operatorname{MSE}(\boldsymbol{x}, \boldsymbol{f})=10 \log \left(\frac{\sum_{j}^{M}\left(\boldsymbol{x}_{j}-\boldsymbol{f}_{j}\right)^{2}}{\sum_{j}^{M}\left(\boldsymbol{f}_{j}\right)^{2}}\right)$, the ensemble mean square bias, Bias $=\frac{\sum_{j}^{M}\left(\boldsymbol{x}_{j}-\boldsymbol{f}_{j}\right)}{\sum_{j}^{M}\left(\boldsymbol{f}_{j}\right)}$, the standard deviation, $\operatorname{Var}=\frac{1}{S} \frac{\left.\sum_{i}^{S} \sum_{j}^{M}\left(\boldsymbol{x}_{j}^{i}-\frac{1}{S} \sum_{i}^{S} \boldsymbol{x}_{j}^{i}\right)\right)^{2}}{\sum_{j}^{M} \boldsymbol{f}_{j}^{2}}$, and the contrast recovery coefficient, CRC $=\frac{1}{S} \sum_{i=1}^{S}\left(\frac{\overline{\boldsymbol{x}}_{\text {esion }}^{i}}{\overline{\boldsymbol{x}}_{B G}^{i}}-\right.$ 1) $/\left(\frac{\bar{f}_{l e s i o n}}{\bar{f}_{B G}}-1\right)$ were used for the quantitative performance evaluation and comparison of the algorithms. In these metrics, $\boldsymbol{x}_{j}$ and $\boldsymbol{f}_{j}$ are respectively the $j$ th elements of the reconstructed and true PET images, $\overline{\boldsymbol{x}}_{\text {lesion }}^{i}, \overline{\boldsymbol{x}}_{B G}^{i}, \overline{\boldsymbol{f}}_{\text {lesion }}$ and $\overline{\boldsymbol{f}}_{B G}$ are respectively the mean pixel values of the reconstructed lesion, reconstructed background (white matter) area, true lesion and true background area, and $S$ is the total number of noisy realizations.

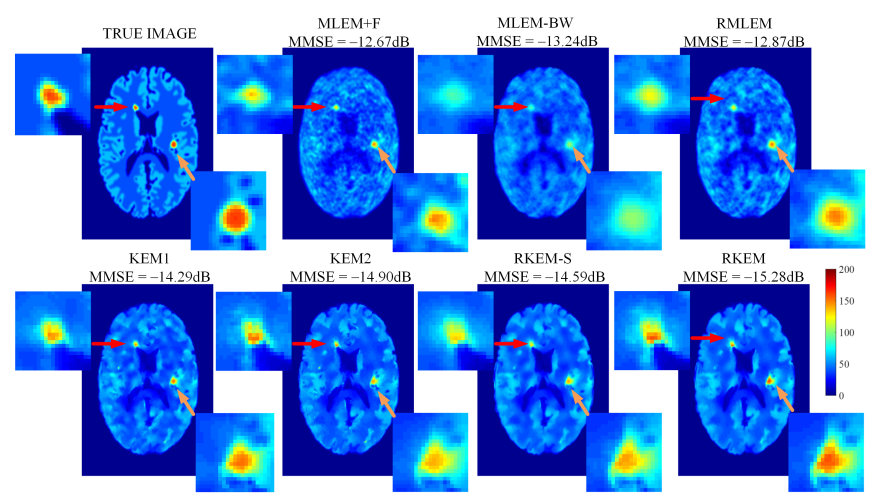

Fig. 3. Reconstruction results from simulated low-count (486k) data.

\section{Image Reconstruction Performance}

Fig. 3 shows the true (reference) image and the images reconstructed by different algorithms from the simulated low count (486k) sinogram data. As seen from these images, all the kernelized methods (second row) outperform their nonkernelized counterparts (first row) in preserving the intensities of lesion pixels and suppressing the whole image noise (measured by MMSE). Of all these images, RKEM image appears to be less noisy, higher contrast and clearer texture, with the pixel intensities (brightness) of lesion areas closer to those of the true image.

Compared with its counterparts without the composite regularizer, RKEM (RMLEM) with the kernel (image) space composite regularizer $V(\boldsymbol{a})(V(\boldsymbol{x}))$ significantly enhances the

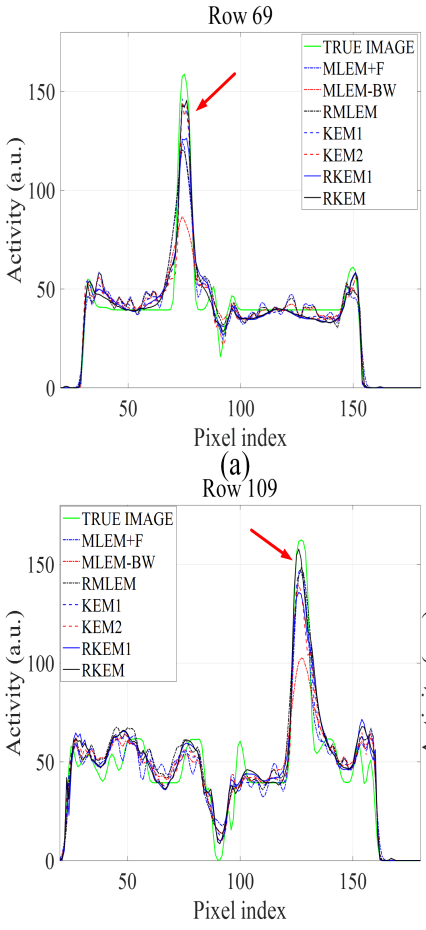

(c)

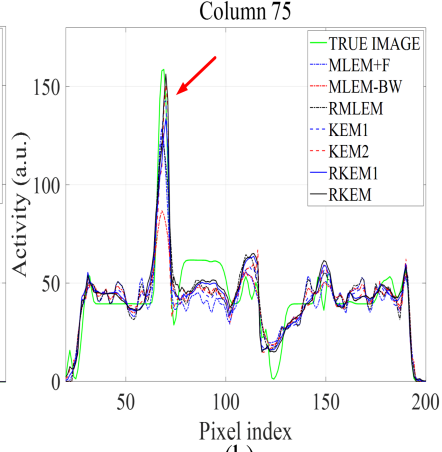

(b)

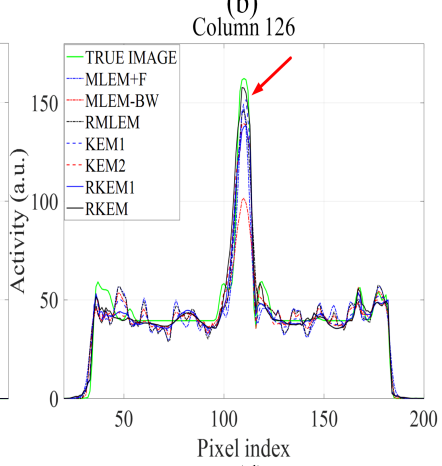

(d)
Fig. 4. Pixel line profile plots across the lesions in low-count images. (a)-(b): Lesion 1. (c)-(d): Lesion 2.

uptakes in lesion areas while suppressing the noise. The uptake enhancement is due to the kernel (image) space energy regularizer $v_{2}(\boldsymbol{a})\left(v_{2}(\boldsymbol{x})\right)$ that promotes the total energy of kernel coefficients $\boldsymbol{a}$ (image $\boldsymbol{x}$ ). While the simultaneous noise suppression is due to the kernel (image) space graph regularizer $v_{1}(\boldsymbol{a})\left(v_{1}(\boldsymbol{x})\right)$ that smoothes the kernel coefficient $\boldsymbol{a}$ (image $\boldsymbol{x})$. Among the three non-kernelized methods compared, RMLEM performs the best in simultaneous uptake enhancement and noise suppression. Compared with KEM1 that was tuned to have KEM's best lesion preservation, RKEM-S with only the kernel space graph regularizer $v_{1}(\boldsymbol{a})$ effectively suppresses the background noise of KEM1 image, but it also reduces the uptakes in lesion areas. Hence RKEM-S image is similar to that of KEM2 which was tuned to have KEM's best noise suppression. In contrast, RKEM avoids the problem of RKEM$\mathrm{S}$ and performs the best among the regularized methods (MLEM-BW, RMLEM, RKEM-S, RKEM) in both lesion preservation and noise suppression. These results demonstrate the individual effects of $v_{1}(\boldsymbol{a})\left(v_{1}(\boldsymbol{x})\right)$ and $v_{2}(\boldsymbol{a})\left(v_{2}(\boldsymbol{x})\right)$, and the advantages of composite regularizer $V(\boldsymbol{a})(V(\boldsymbol{x}))$.

For quantitative comparison, Fig. 4 plots the pixel line profiles across the lesions in the low count images of Fig. 3. The lesion area shows up as one peak indicated by the red arrow, which is significantly different from the white matter area in the low count image. As seen from the plots, RKEM yields the highest uptakes in MR-PET Lesion 1 and PETonly Lesion 2, which are closer to those of the true image as compared with other algorithms.

Fig. 5 shows the images reconstructed by different algorithms from the simulated high count (1133k) sinogram 
data. Due to the high count of this image, all algorithms achieve better results. RKEM images are sharper and less noisy with lower MMSE and globally clearer image details than other images. The lesion areas in RKEM image are more pronounced than in other images. For quantitative comparison, Fig. 6 plots the pixel line profiles across the lesions in the high count images of Fig. 5. As seen from the plots, RKEM's uptakes in Lesion 1 and Lesion 2 are closer to those of the truth image as compared with those of other algorithms.

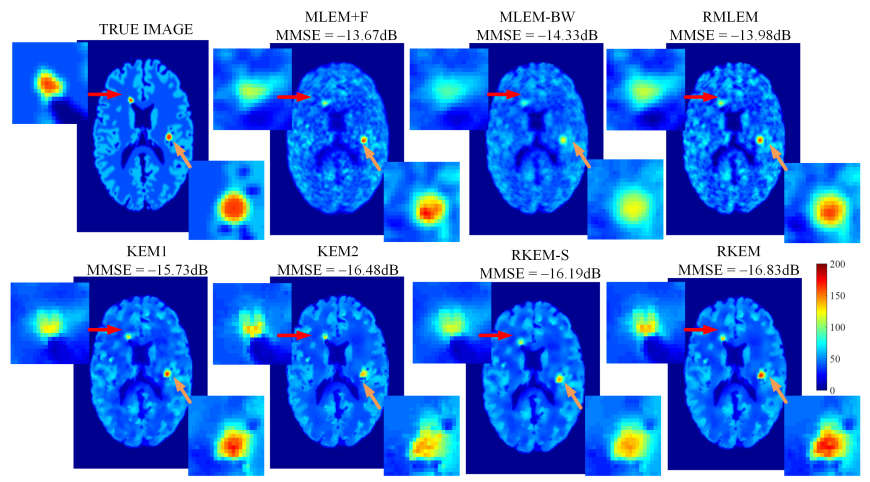

Fig. 5. Reconstruction results from simulated high-count (1133k) data.

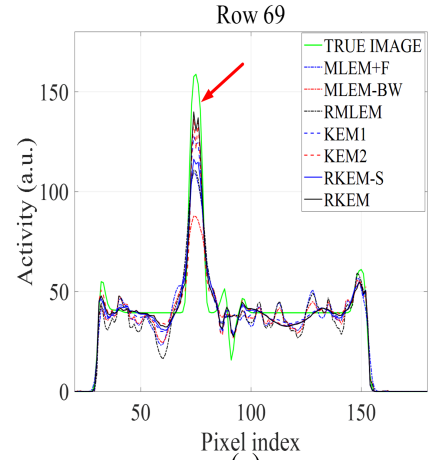

(a)

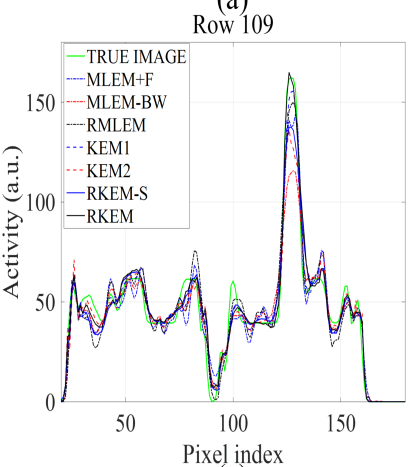

(c)

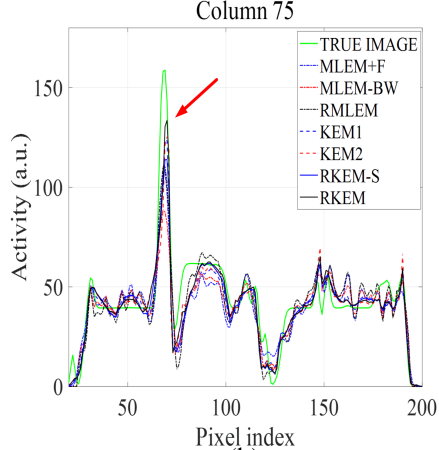

(b)

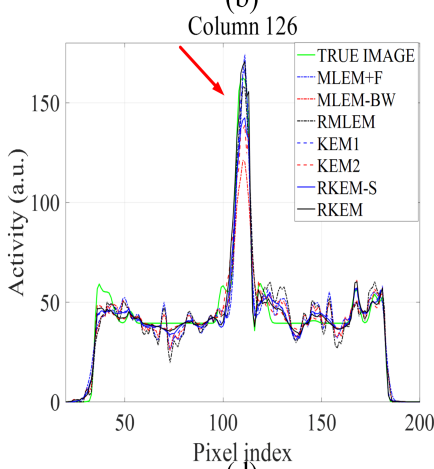

(d)
Fig. 6. Pixel line profile plots across the lesions in high-count images. (a)-(b): Lesion 1. (c)-(d) Lesion 2.

\section{AMSE, bias vs variance, $C R C$ vs background noise}

Fig. 7 plots the average MSE (AMSE) versus the iteration number of different methods over ten noisy realizations. As seen from the plots, initially, all the methods approach their respective minimum AMSE's as the iteration number increases,

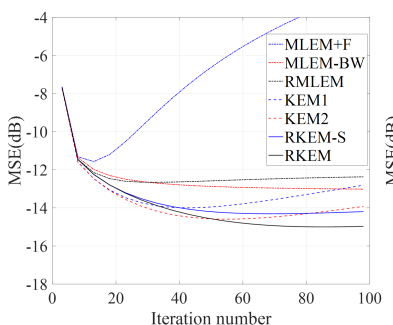

(a)

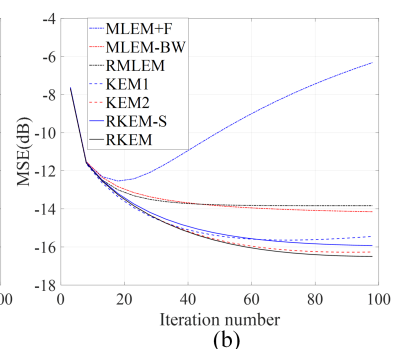

(b)
Fig. 7. Average MSE versus iteration number of different algorithms for (a) low count image and (b) high count image.

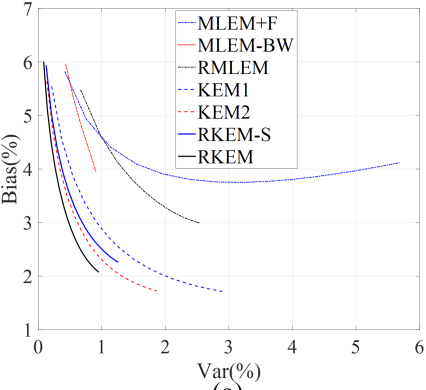

(a)

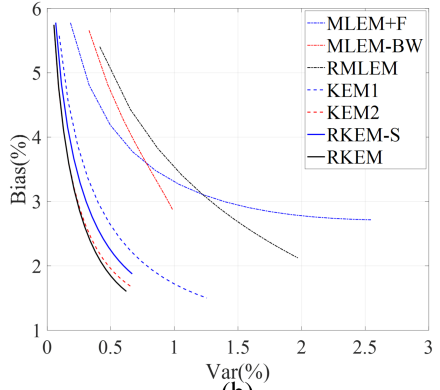

(b)
Fig. 8. Ensemble mean squared bias versus mean variance trade-off of different algorithms over iterations 1 to 100 in increments of 5. (a) Low count image and (b) high count image.

with the minimum AMSE of RKEM being the lowest among all the methods. When the iteration number further increases to beyond the minimum AMSE points, the AMSE's of unregularized methods start to increase due to their lack of noise suppression regularizer, and the increases are rather fast in low count image. Whereas the AMSE's of regularized methods tend to settle down, and that of RKEM settles down at the lowest value monotonically, with little change as iterations further increase.

Fig. 8 shows the ensemble mean squared bias versus the mean variance trade-off of different algorithms over the iterations 1 to 100 in the increments of 5. As seen from the figure, the proposed RKEM always get much lower bias and variance simultaneously than those of other methods for both low count and high count images.

Fig. 9 plots CRC versus the standard deviation of background noise of different methods for Lesion 1 and Lesion 2 , over the iterations 1 to 100 in the increments of 5 . As seen from the figure, RKEM attains higher CRC with lower background noise than those of other methods.

\section{E. Effects of parameters and prior data sources}

Fig. 10 shows the effect of regularization parameter $\beta$ on the low count image quality of RKEM. With the fine-tuned optimal $\beta=4 \times 10^{-3}$, RKEM attains the best tradeoff between the uptakes in two lesion areas and the clearness of image texture as shown in Fig. 10(b). When $\beta$ is tuned to below the optimal value at $\beta=4 \times 10^{-4}$, the kernel space graph regularizer $v_{1}(\boldsymbol{a})$ and the kernel space energy regularizer $v_{2}(\boldsymbol{a})$ are both weighted down. As a result, the 


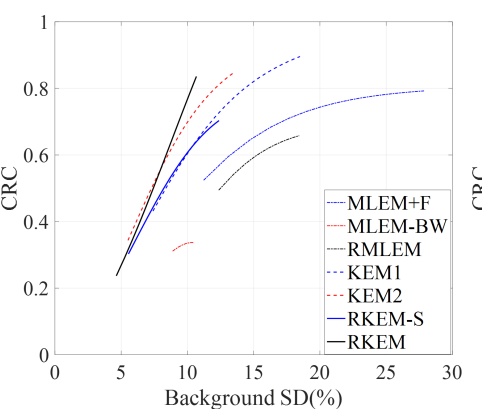

(a)

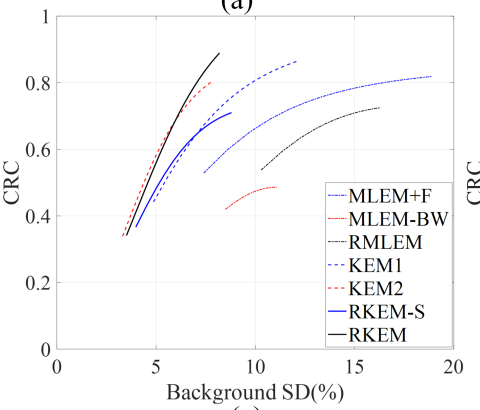

(c)

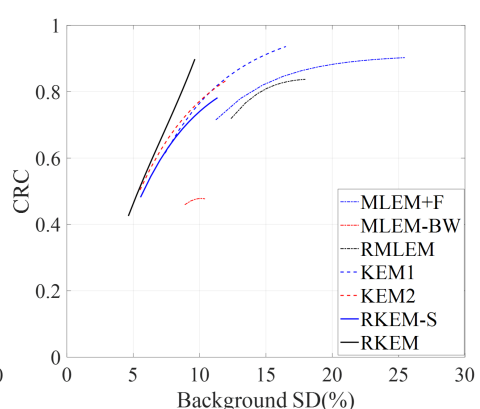

(b)

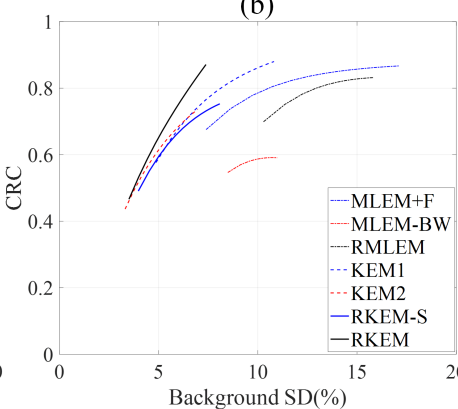

(d)

Fig. 9. $\mathrm{CRC}$ of lesion regions versus standard deviation of background noise over iterations 1 to 100 in increments of 5. (a) Lesion 1 in low count image. (b) Lesion 2 in low count image. (c) Lesion 1 in high count image. (d) Lesion 2 in high count image.

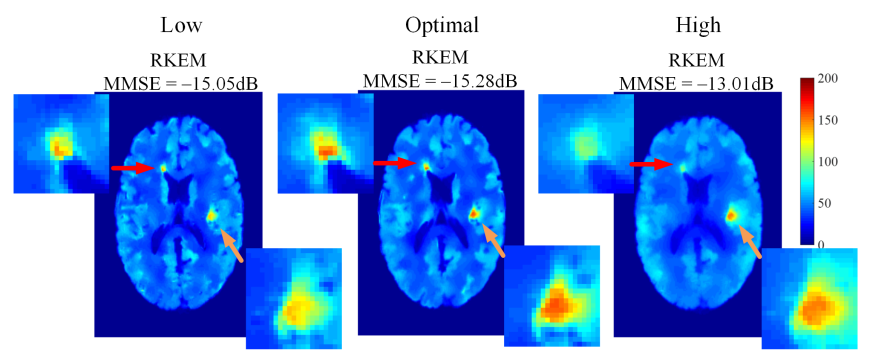

Fig. 10. Effect of regularization coefficient $\boldsymbol{\beta}$ on RKEM image quality

image texture, as shown in Fig. 10(a), becomes slightly sharper due to decreased smoothing effect of $v_{1}(\boldsymbol{a})$, also, the uptakes in two lesion areas become lower due to decreased pixel energy enhancement of $v_{2}(\boldsymbol{a})$. When $\beta$ is tuned to above the optimal value at $\beta=4 \times 10^{-2}, v_{1}(\boldsymbol{a})$ and $v_{2}(\boldsymbol{a})$ are both weighted up. Hence, the image texture, as shown in Fig. 10(c), becomes blurred due to stronger smoothing effect of $v_{1}(\boldsymbol{a})$, which also smoothes out the energy of Lesion 1 and makes it nearly invisible. But the uptake in PET-only Lesion 2 is well kept by the stronger energy enhancement of $v_{2}(\boldsymbol{a})$ that is derived from the pre-reconstructed PET image with the information on Lesion 2. These results show that the regularization parameter $\beta$ needs to be tuned properly. If it is too high or too low, the image quality of RKEM may degrade.

As detailed in Subsections II-C and II-E, the kernel space composite regularizer $V(\boldsymbol{a})$ is derived from the kernel matrix $\boldsymbol{K}^{O}$, which can be constructed from the prior image data of different sources. To show the impact of different sources, Fig. 11 compares the images reconstructed by RKEM with $V(\boldsymbol{a})$ derived from MR prior image data, named RKEM-MR, and

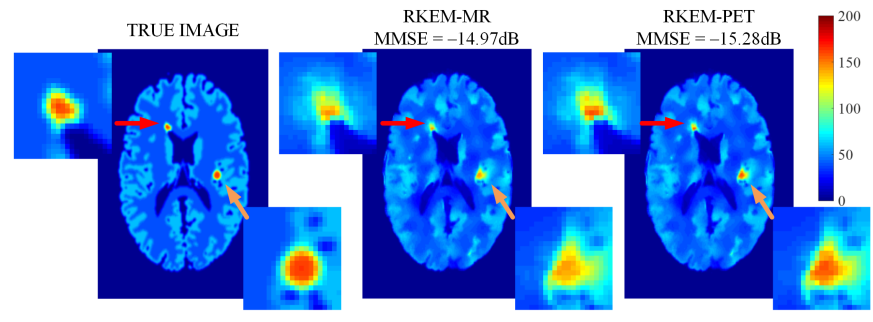

Fig. 11. Effect of prior data from different sources.

from pre-reconstructed PET prior image data, named RKEMPET. As seen from the figure, the uptake of PET-only lesion in RKEM-MR images is lower due to the lack of PET-only lesion information in the $V(\boldsymbol{a})$ used in RKEM-MR. Besides the source of prior image data, the neighbourhood size $J$ and kernel parameter $\sigma$ are also selectable in $\boldsymbol{K}^{O}$ construction. We found in our experiments that the $J$ and $\sigma$ of $\boldsymbol{K}^{O}$ were well aligned with those of the kernel matrix $\boldsymbol{K}$ in KEM. The $J$ and $\sigma$ good for $\boldsymbol{K}$ are also good for $\boldsymbol{K}^{O}$. Hence, we used the $J$ and $\sigma$ of KEM2 for RKEM-S and RKEM in our experiments.

\section{TESTS ON IN-VIVO DATA}

To validate the results of simulation studies and further compare the performance of MLEM+F, MLEM-BW, RMLEM, KEM1, KEM2 and RKEM, we have applied them to 2D in-vivo human data. Their settings and parameters were the same as those in simulation studies, except the regularization parameter of RKEM and RMLEM was tuned to $\beta=4 \times 10^{-5}$. We have also tested a new RKEM1, with KEM1 regularized by the same kernel space composite regularizer $V(\boldsymbol{a})$ of RKEM. For symbol consistence, we rename RKEM as RKEM2 in the sequel.

The PET in-vivo human data and corresponding MR image were acquired from a Siemens (Erlangen) Biograph 3 Tesla molecular MR (mMR) scanner. The human PET data were acquired with $233 \mathrm{MBq}$ of [18-F]FDG infusion over the course of 95 minutes at the rate of $36 \mathrm{~mL} / \mathrm{hr}$ and the average concentration about $2.45 \mathrm{MBq} / \mathrm{min}$ (corrected to the acquisition start time). The 95 minutes list-mode data were binned into 95 3D sinogram frames each of 1-minute. A pseudo CT attenuation map was generated from the UTE map and was used to correct attenuation for acquired data [39]. T1 weighted MR image data were acquired at the scan time 30 minutes from a 16channel RF head coil, with scan parameters $\mathrm{TA}=7.01 \mathrm{mins}$, $\mathrm{TR}=1640 \mathrm{~ms}$, TE $=2.34 \mathrm{~ms}$, flip angle $=8$ degrees, FOV $=256 \times 256 \mathrm{~mm}^{2}$, voxel size $=1 \times 1 \times 1 \mathrm{~mm}^{3}, 176$ slices. For details of data acquisition and preprocessing, see [40].

The raw data of a slice of PET images at the 50 minutes of scan was rebinned into sinograms with 157 angular projections and 219 radial bins, which were used for testing PET image reconstruction. MLEM with 20 iterations and post Gaussian filtering was applied to the sinograms to obtain the PET prior image for constructing the kernel matrix $\boldsymbol{K}^{O}$. The T1 weighted MR image was used as the prior image data to construct the kernel matrix $\boldsymbol{K}$.

Fig. 12 shows the MR prior image and images reconstructed by the compared five methods. The following can be observed 


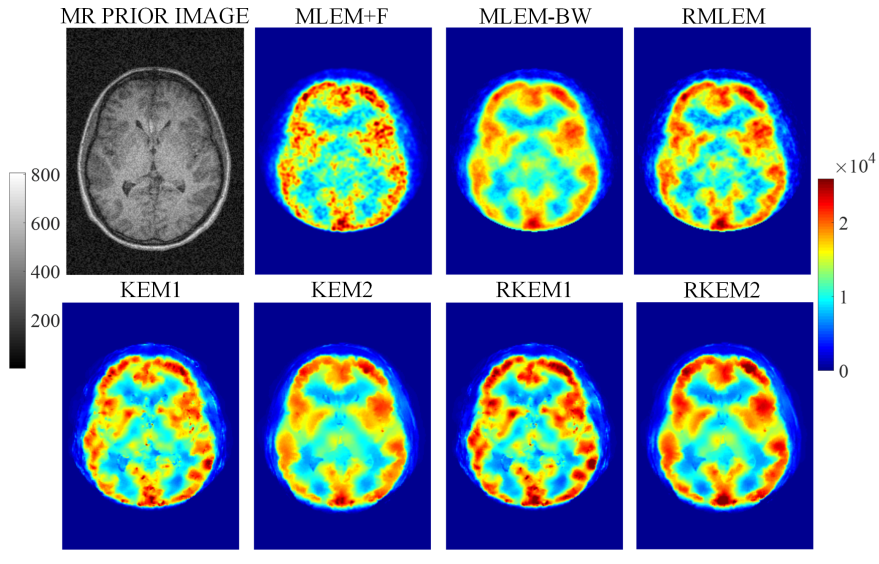

Fig. 12. Reconstruction results from in-vivo data

from careful comparison of these images: i) MLEM+F image appears noisy with pronounced granularity, especially in the areas with high uptakes. ii) Equipped with the conventional $\mathrm{BW}$ regularizer, MLEM-BW smoothes out the image globally, which significantly reduces granularity everywhere, but also reduces uptakes in some areas rather significantly. iii) Having the new image space composite regularizer $V(\boldsymbol{x})$ derived from PET prior image, RMLEM effectively suppresses granularity with little loss of uptakes, but its image texture appears less smooth than that of MLEM-BW image and closer to that of MLEM+F image. This is because $V(\boldsymbol{x})$ was derived from PET prior image pre-reconstructed by MLEM+F. iv) With the kernel matrix constructed from MR prior image and without a regularizer, KEM1 and KEM2 smooth out the image globally without much loss of uptakes. KEM1 yields sharper texture with more details because its $J=11$ and $\sigma=0.15$ were tuned to achieve the best preservation of small objects (which was PET-only lesion in simulation studies). KEM2 image appears smoother than that of KEM1 with less texture details because its $J=19$ and $\sigma=0.25$ were tuned to achieve the best noise suppression. v) Equipped with the kernel matrix constructed from MR prior image and the composite kernel space regularizer $V(\boldsymbol{a})$ derived from PET prior image, RKEM1 and RKEM2 significantly smooth out the image and enhance the uptakes everywhere. RKEM1 image appears sharper with more texture details than RKEM2 image due to the difference of KEM1 and KEM2 in $J$ and $\sigma$. Among the images of all methods, RKEM1 and RKEM2 images appear to have smoother texture, higher uptakes and contrast, and more pronounced anatomical structures, though it is impossible to judge which image is the best since no ground truth available.

\section{Discussions}

As seen from Fig. 7, KEM may behave similarly to MLEM with its AMSE increasing beyond the lowest value along with iterations, especially in low count reconstruction, but the AMSE and the increase rate are much lower than those of MLEM due to the smoothing effect of kernel matrix $\boldsymbol{K}$. These are consistent with the result of [11] and demonstrate the need for regularized KEM - RKEM.
The simulation and in vivo data tests have shown that RKEM outperforms MLEM+F, MLEM-BW, RMLEM and KEM, producing visually better PET images with clearer texture and higher uptakes and contrast, lower AMSE, monotonic convergence, insensitivity to iteration number, simultaneously lower bias and variance, and higher CRC with lower background noise. These results clearly justify the use of RKEM to enhance KEM.

The better performance of RKEM stems from the four ingredients of the kernel space composite regularizer $V(\boldsymbol{a})$. 1) The kernel space graph regularizer $v_{1}(\boldsymbol{a})$ smoothes the kernel coefficient $\boldsymbol{a}$, which in turn smoothes out the reconstructed image $\hat{\boldsymbol{x}}$ to reduce image noise. 2) The kernel space energy regularizer $v_{2}(\boldsymbol{a})$ promotes the total energy of kernel coefficients $\boldsymbol{a}$, which in turn promotes the uptakes and small objects, such as lesions, of the image. 3) The composition constant $\gamma$ set by (16) guarantees $V(\boldsymbol{a})$ 's convexity, which in turn leads to RKEM's monotonic convergence and insensitivity to the iteration number. 4) The PET prior image data used to derive $V(\boldsymbol{a})$ contains PET-only information, which allows RKEM to better preserve PET image features such as PET-only lesions.

The above advantages are also observed from RMLEM. With the non-kernelized image space composite regularizer $V(\boldsymbol{x})$, RMLEM can also suppress image noise while enhancing PET-only lesion, though the resulting image is not as good as RKEM image due to the non-kerneled MLEM.

Although $V(\boldsymbol{a})$ has four ingredients, its strength is controlled by a single regularization parameter $\beta$, which is the same as in conventional RML (MAP) optimization (3), without additional hyper-parameter.

KEM's performance depends on its neighbourhood size $J$ and kernel parameter $\sigma$. We only used the nearest pixels as $J$ and followed the literature, eg [2], to tune $J$ and $\sigma$ in our experiments. We have not used other methods, such as the $k \mathrm{NN}$ method [2], to determine $J$ for simplicity. As RKEM uses the kernel space composite regularizer $V(\boldsymbol{a})$ to regularize KEM, its effects are not critically affected by KEM's parameters, provided KEM itself is reasonably tuned.

For simpler and clearer demonstration of RKEM's essence, we have focused on 2D PET image reconstruction in our simulation studies and in vivo data tests. With some minor modifications in algorithm setup, similar results can be obtained in $3 \mathrm{D}$ case.

\section{CONCLUSION}

This paper has presented a novel kernel space regularization method, called RKEM, for the recently emerged KEM method to avoid its possibility of large reconstruction error and high sensitivity to iteration number, and to enhance its image quality.

To overcome the difficulty in developing the globally convergent optimization algorithm for RKEM, we have used the concept of data manifolds and the technique of graph regularization to develop the kernel space graph regularizer, the kernel space energy regularizer and their composition, the kernel space composite regularizer. Combining the kernel space composite regularizer with the optimization transfer 
technique, we have presented a globally convergent RKEM algorithm for PET image reconstruction. A special case of this algorithm, dubbed RMLEM, is a globally convergent optimization algorithm for the conventional (non-kernelized) regularized ML (MAP) reconstruction, but with a new image space composite regularizer for better performance.

The four ingredients of the kernel space composite regularizer, as discussed in Section V, are the power source of the proposed RKEM, which sets RKEM apart from the exiting kernelized methods and empowers it with better performance.

The results of simulation studies and in vivo data tests have shown the better performance of the proposed RKEM over KEM and other conventional iterative methods in PET image reconstruction. These results have also shown the better performance of RMLEM over conventional iterative reconstruction methods.

\section{REFERENCES}

[1] W. Hutchcroft, G. B. Wang, and K. T. Chen, "Anatomically-aided PET reconstruction using the kernel method," Physics in Medicine \& Biology, vol. 61, no. 18, pp. 66-68, 2016

[2] G. B. Wang, J. Qi, "PET image reconstruction using kernel method," IEEE Transactions on Medical Imaging, no. 34, vol. 1, pp. 61-71, 2015.

[3] J. Bland et al. "MR-guided kernel EM reconstruction for reduced dose PET imaging," IEEE Transactions on Radiation and Plasma Medical Sciences, no. 2, vol. 3, pp. 235-243, 2018.

[4] P. Novosad and A. J. Reader, "MR-guided dynamic PET reconstruction with the kernel method and spectral temporal basis functions," Physics in Medicine \& Biology, no. 61, vol. 12, pp. 4624-4645, 2016.

[5] K. Gong, J. Cheng-Liao, G. B. Wang, K. T. Chen, C. Catana, and J. Qi, "Direct Patlak reconstruction from dynamic PET data using kernel method with MRI information based on structural similarity," IEEE Transactions on Medical Imaging, no. 37, vol. 4, pp. 955-965, 2018.

[6] J. Bland, M. Belzunce, S. Ellis, C. McGinnity, and A. Hammers, "Spatially-compact MR-guided kernel EM for PET image reconstruction," IEEE Transactions on Radiation and Plasma Medical Sciences, vol. 2, no. 5, pp. 470-482, 2018.

[7] S. Ellis and A. Reader, "Kernelised EM image reconstruction for dual-dataset PET studies," IEEE Nuclear Science Symposium, Medical Imaging Conference and Room-Temperature Semiconductor Detector Workshop, Strasburg, France, pp. 1-3, 2016.

[8] R. Baikejiang, Y. Zhao, B. Z. Fite, K. W. Ferrara, and C. Q. Li, "Anatomical image guided fluorescence molecular tomography reconstruction using kernel method," Journal of Biomedical Optics, no. 22, vol. 5, pp. 055-067, 2017.

[9] B. Spencer and G. B. Wang, "Statistical image reconstruction for shortened dynamic PET using a dual kernel method," in Proceedings of NSS/MIC, Atlanta, GA, USA, September, 2017.

[10] J. Qi and R. M. Leahy, "Iterative reconstruction techniques in emission computed tomography," Physics in Medicine \& Biology, no. 51, vol. 15, pp. R541, 2006

[11] G. B. Wang, "High temporal-resolution dynamic PET image reconstruction using a new spatiotemporal kernel," IEEE Transactions on Medical Imaging, no. 38, vol. 3, pp. 664-674, 2019

[12] M. J. Ehrhardt et al. "PET reconstruction with an anatomical MRI prior using parallel level sets," IEEE Transactions on Medical Imaging, vol. 35, pp. 2189-2199, 2016.

[13] K. Vunckx et al. "Evaluation of three MRI-based anatomical priors for quantitative PET brain imaging," IEEE Transactions on Medical Imaging, vol. 31, pp. 599-612, 2012.

[14] J. Tang, Y. Wang, R. Yao, and L Ying, "Sparsity-based PET image reconstruction using MRI learned dictionaries," International Symposium on Biomedical Imaging, Beijing, China, April, 2014, pp. 1087-1090.

[15] M. N. Wernick and J. N. Aarsvold, "Emission tomography: the fundamentals of PET and SPECT," Elsevier, 2004.

[16] E. Levitan and G. T. Herman, "A maximum a posteriori probability expectation maximization algorithm for image reconstruction in emission tomography," IEEE Transactions on Medical Imaging, vol. 6, no. 3, pp. 185-192, 1987.
[17] P. J. Green, "Bayesian reconstructions from emission tomography data using a modified EM algorithm," IEEE Transactions on Medical Imaging, vol. 9, no. 1, pp. $84-93,1990$.

[18] D. Lalush and B. Tsui, "Simulation evaluation of Gibbs prior distributions for use in maximum a posteriori SPECT reconstructions," IEEE Transactions on Medical Imaging, no. 11, vol. 2, pp. 267-275, 1992.

[19] A. R. De Pierro, "A modified expectation maximization algorithm for penalized likelihood estimation in emission tomography," IEEE Transactions on Medical Imaging, no. 14, vol. 1, pp. 132-137, 1995.

[20] A. P. Dempster, N. M. Laird, and D. B. Rubin, "Maximum likelihood from incomplete data via the EM algorithm," J. R. Stat. Soc. Series B, vol. 39 , no. 1 , pp. 138,1977

[21] K. Lange, D. R. Hunter, and I. Yang, "Optimization transfer using surrogate objective functions," Journal of Computational and Graphical Statistics, no. 9, vol. 1, pp. 1-20, 2000.

[22] K. Gong, C. Catana, J. Qi and Q. Li, et al. "PET image reconstruction using deep image prior," IEEE Transactions on Medical Imaging, no. 38, vol. 7, pp. 1655-1665, 2018.

[23] G. B. Wang and J. Qi, "Penalized likelihood PET image reconstruction using patch-based edge-preserving regularization," IEEE Transactions on Medical Imaging, no. 31, vol. 12, pp. 2194-2204, 2012.

[24] A. J. Smola and R. Kondor, "Kernels and Regularization on Graphs," Learning Theory and Kernel Machines, 2003.

[25] M. Belkin and P. Niyogi, "Laplacian eigenmaps for dimensionality reduction and data representation," Neural Computation, no. 15, vol. 6, pp. 13731396, 2003.

[26] M. Zheng et al. "Graph regularized sparse coding for image representation," IEEE Transactions on Image Processing, no. 20, vol. 5, pp. 1327-1336, 2010.

[27] Y. Yankelevsky and M. Elad, "Dual graph regularized dictionary learning," IEEE Transactions on Signal and Information Processing over Networks, no. 2, vol. 4, pp. 611-624, 2016.

[28] M. Elad, "Sparse and redundant representations from theory to Aapplications in signal and image processing," Springer, 2010.

[29] R. Sinkhorn, and P. Knopp, "Concerning nonnegative matrices and doubly stochastic matrices," Pacific Journal of Mathematics, no. 21, vol. 2, pp. 343-348, 1967.

[30] A. Kheradmand and P. Milanfar, "A general framework for regularized, similarity-based image restoration," IEEE Transactions on Image Processing, no. 23, vol. 12, pp. 5136-5151, 2014.

[31] A. Y. Ng, M. Jordan, and Y. Weiss, "On spectral clustering: Analysis and an algorithm," in Advances in Neural Information Processing Systems, Vancouver, Canada, 2001, pp. 849-856.

[32] J. Liu, D. Cai, and X. He, "Gaussian mixture model with local consistency," in Twenty-Fourth Conference on Artificial Intelligence, 2010, no. 1, vol. 24.

[33] M. Belkin, P. Niyogi, and V. Sindhwani, "Manifold regularization: A geometric framework for learning from labeled and unlabeled examples," The Journal of Machine Learning Research, vol. 7, pp. 23992434, 2006.

[34] D. Zhou, O. Bousquet, T. Lal, J. Weston, and B. Scholkopf, "Learning with local and global consistency," in Advances in Neural Information Processing Systems, 2003, pp. 321-328.

[35] S. Boyd, S. P. Boyd, L. Vandenberghe, "Convex optimization," Cambridge university press, 2004.

[36] J. E. Bowsher et al., "Utilizing MRI information to estimate F18-FDG distributions in rat flank tumors," IEEE Nuclear Science Symposium Conference Record, 2004, pp. 2488-2492.

[37] C.A. Cocosco, V. Kollokian, R. K. S. Kwan, and A.C. Evans, "BrainWeb: Online interface to a 3D MRI simulated brain database," Proceedings of 3rd International Conference on Functional Mapping of the Human Brain. Copenhagen, Denmark, May, 1997.

[38] D. L. Collins et al. "Design and construction of a realistic digital brain phantom," IEEE Transactions on Medical Imaging, vol. 17, no. 3, pp. 463-468, 1998.

[39] N. Burgos et al. "Attenuation correction synthesis for hybrid PET-MR scanners: application to brain studies", IEEE Transactions on Medical Imaging, vol. 33, pp. 2332-2341, 2014.

[40] S. D. Jamadar, P. G. Ward, E. X. Liang, E. R. Orchard, Z. Chen, and G. F Egan, "Metabolic and haemodynamic resting-state connectivity of the human brain: a high-temporal resolution simultaneous BOLD-fMRI and FDG-fPET multimodality study," Cerebral Cortex, no. 31 vol. 6, pp. $2855-2867,2021$. 